\title{
Gast-Editorial ZfOU - Kongressheft
}

\author{
Liebe Kolleginnen und Kollegen, \\ $\nabla$
}

"Menschen bewegen - Erfolge erleben"

Dies ist das Motto unseres Jahreskongresses 2013.

Orthopäden und Unfallchirurgen bewegen Menschen im doppelten Sinne des Wortes. Wir behandeln, heilen oder lindern Erkrankungen und Verletzungen des muskuloskelettalen Systems sowie Polytraumen. Damit ermöglichen wir unseren Patienten wieder eine schmerzfreie Bewegung, Teilhabe und Lebensqualität. Dies sind unbestreitbare Erfolge für uns - und vor allem für unsere Patienten. Das wird von einer breiten Öffentlichkeit und der Gesundheitspolitik auch erwartet und anerkannt - zunehmend und immer häufiger aber auch infrage gestellt. Wir bewegen daher auch eine mediengesteuerte Diskussion um Behandlungsqualität, Indikationsstellungen und Mittelzuteilung.

Dieser Diskussion stellen wir uns gerne und werden auf unserem Kongress unsere wissenschaftlichen und berufspolitischen Themenschwerpunkte wieder selbstkritisch, aber auch sehr selbstbewusst gemeinsam bearbeiten und öffentlich vertreten. Wir, das sind Ihre Kongresspräsidenten 2013, meine Kollegen Prof. Dr. Bernd Kladny für die DGOOC und Prof. Dr. Karl-Dieter Heller für den BVOU sowie ich selbst für die DGU und als Präsident der DGOU.

Die DGOU feiert in 2013 ein kleines - nämlich ihr fünfjähriges - Jubiläum. Für diesen kurzen Zeitraum eine beeindruckende Erfolgsgeschichte mit stetig wachsenden Mitgliederzahlen und mittlerweile 18 eigenständigen Sektionen. Hierbei haben sich ganze wissenschaftliche Fachgesellschaften in die DGOU als Sektionen eingebracht. Dies zeigt zum einen die Anerkennung der Notwendigkeit einer gemeinsamen und strukturierten Dachorganisation für $\mathbf{O}$ und $\mathbf{U}$ und ist gleichzeitig aber auch Ausdruck einer fortschreitenden Spe- zialisierung. Nicht zuletzt drückt sich hierin ein gemeinsames Bestreben aus, Synergien zu schaffen und zu stärken sowie Doppelstrukturen möglichst zu vermeiden. Ein Anliegen, dass für unser Fach auch im Sinne einer ganzheitlichen Außendarstellung unverzichtbar ist.

Kernaufgaben wissenschaftlicher Fachgesellschaften sind die Unterstützung und Förderung seriöser wissenschaftlicher Grundlagenforschungstätigkeit und die Durchführung strukturiert geplanter klinischer Studien - und dies womöglich prospektiv-randomisiert und multizentrisch. Das stellt uns zunehmend vor immense Herausforderungen. Unsere Leistungsfähigkeit als Fachgesellschaft hängt wesentlich vom Engagement unserer Mitglieder und von deren Möglichkeiten ab. Schaffen Sie sich daher Freiräume und werden Sie aktiv - in der DGOU. Organisieren Sie sich in den etablierten klinischen und Forschungsnetzwerken von $\mathrm{O}$ und $\mathrm{U}$. Gemeinsam erreichen wir mehr! Die deutsche Orthopädie und Unfallchirurgie wird ihre Spitzenstellung zukünftig nur behaupten können, wenn auch weiterhin aktiv und erfolgreich geforscht und publiziert wird.

Die ZfOU stellt hierbei für den deutschsprachigen Raum ein erfolgreiches Publikationsorgan für unser gemeinsames Fachgebiet dar. Sie hat sich nach ihrem Start rasch „am Markt“ etabliert und wird durch den stetigen Eingang qualitativ hochwertiger Manuskripte und deren Zitierungen auch zukünftig weiter an Bedeutung gewinnen. Auch hier sind Sie herzlich eingeladen, sich aktiv einzubringen und Ihre Ergebnisse zu präsentieren.

Ich wünsche Ihnen in diesem Sinne auch zukünftig viele Freude und Erfolg für Ihre Tätigkeit in 0 und $\mathrm{U}$ !
Herzlichst
Ihr
Reinhard Hoffmann

DOI http://dx.doi.org/ 10.1055/s-0033-1350695

Z Orthop Unfall 2013; 151: 337

(c) Georg Thieme Verlag KG

Stuttgart · New York .

ISSN 1864-6697

\author{
Korrespondenzadresse \\ Prof. Dr. med. \\ Reinhard Hoffmann \\ Präsident DGU 2013 \\ Präsident DGOU 2013 \\ Ärztlicher Geschäftsführer und \\ Ärztlicher Direktor \\ Berufsgenossenschaftliche \\ Unfallklinik Frankfurt am Main \\ Friedberger Landstraße 430 \\ 60389 Frankfurt am Main \\ Tel.: 069/475-2001 \\ aerztlicher.direktor@ \\ bgu-frankfurt.de
}

